\title{
Infinite Paths in Planar Graphs I, Graphs with Radial Nets
}

\author{
Xingxing $\mathrm{Yu}$ * \\ School of Mathematics \\ Georgia Institute of Technology \\ Atlanta, Georgia 30332
}

May 14, 2002

\begin{abstract}
Let $G$ be an infinite 4-connected planar graph such that the deletion of any finite set of vertices from $G$ results in exactly one infinite component. Dean et al proved that either $G$ admits a radial net or a special subgraph of $G$ admits a ladder net, and they used these nets to show that $G$ contains a spanning 1-way infinite path. In this paper, we show that if $G$ admits a radial net, then $G$ also contains a spanning 2 -way infinite path. This is a step towards a conjecture of Nash-Williams.
\end{abstract}

${ }^{*}$ Partially supported by NSF grant DMS-9970527 


\section{Introduction and notation}

We consider only simple graphs. Let $G$ be a graph. We use $V(G)$ and $E(G)$ to denote its vertex set and edge set, respectively. If $V(G) \cup E(G)$ is infinite, then $G$ is an infinite graph; otherwise, $G$ is a finite graph. The graphs in this paper could be finite or infinite. We say that $G$ is planar if $G$ can be drawn in the plane with no pair of edges crossing, and such a drawing is called a plane graph or a plane representation of $G$. A graph $H$ is said to be a subgraph of $G$, denoted by $H \subset G$, if $V(H) \subset V(G)$ and $E(H) \subset E(G)$. A graph $H$ is a spanning subgraph of $G$ if $H \subset G$ and $V(H)=V(G)$. For $X \subset V(G)$ and a subgraph $H$ of $G$, let $H-X$ denote the graph obtained from $H$ by deleting $X$ and the edges of $H$ incident with vertices in $X$. We say that $G$ is connected if there is a finite path between any two vertices in $G$. A component of $G$ is a maximal connected subgraph of $G$. We say that $G$ is $k$-connected, where $k$ is a positive integer, if $|V(G)| \geq k+1$ and $G-X$ is connected for any $X \subset V(G)$ with $|X| \leq k-1$. A set $X \subset V(G)$ is a $k$-cut in $G$ if $|X|=k$ and $G-X$ is not connected.

In 1931, Whitney [8] proved that every finite 4-connected planar triangulation contains a Hamilton cycle. In 1956, Tutte [7] proved that every finite 4-connected planar graph contains a Hamilton cycle. It is natural to ask if this result can be extended to infinite graphs. A 1-way infinite path is a graph which is isomorphic to the graph with vertex set $\left\{v_{i}: i=1,2, \cdots\right\}$ and edge set $\left\{v_{i} v_{i+1}: i=1,2, \cdots\right\}$, and a 2-way infinite path is a graph which is isomorphic to the graph with vertex set $\left\{v_{i}: i=\cdots,-2,-1,0,1,2, \cdots\right\}$ and edge set $\left\{v_{i} v_{i+1}: i=\cdots,-2,-1,0,1,2, \cdots\right\}$. From now on, a path could be finite, or 1-way infinite, or 2-way infinite. Nash-Williams ([2], [3], also see [5]) conjectured that an infinite 4-connected planar graph $G$ contains a spanning 1-way infinite path if, and only if, for every finite $X \subset V(G), G-X$ has exactly one infinite component. This conjecture has been verified by Dean, Thomas and Yu [1]. Nash-Williams ([2] and [3]) also made the following conjecture.

(1.1) Conjecture. An infinite 4-connected planar graph $G$ contains a spanning 2-way infinite path if, and only if, for every finite $X \subset V(G), G-X$ has at most two infinite components.

For convenience, we say that a graph $G$ is $k$-indivisible, where $k$ is a positive integer, if, for every finite $X \subset V(G), G-X$ has at most $k-1$ infinite components. It is shown ([1], Theorem (2.3)) that if $G$ is an infinite 2-indivisible 4-connected planar graph then 
either $G$ admits a "radial net" or a special subgraph of $G$ admits a "ladder net". The structures of nets are then used in [1] to find spanning 1-way infinite paths in infinite 2-indivisible 4-connected planar graphs. The main objective of this paper is to prove (1.1) for graphs which admit radial nets.

By the Jordan curve theorem, each cycle $C$ in an infinite plane graph $G$ divides the plane into two closed regions (whose intersection is $C$ ). If exactly one of these two closed regions, say $\mathcal{R}$, contains only finitely many vertices and edges of $G$, then we use $I_{G}(C)$ to denote the subgraph of $G$ consisting of the vertices and edges of $G$ contained in $\mathcal{R}$. Note that $I_{G}(C)$ is a finite subgraph of $G$. If there is no confusion, we use $I(C)$ instead of $I_{G}(C)$. Note that $C \subset I(C)$, and if $I(C)=C$ then $C$ is a facial cycle.

Let $K$ and $H$ be subgraphs of a graph. Then $K \cup H$ (respectively, $K \cap H$ ) denotes the union (respectively, intersection) of $K$ and $H$. Hence, $V(K \cup H)=V(K) \cup V(H)$, $E(K \cup H)=E(K) \cup E(H), V(K \cap H)=V(K) \cap V(H)$, and $E(K \cap H)=E(K) \cap E(H)$.

(1.2) Definition. A radial net in an infinite plane graph $G$ is a sequence $N=\left(C_{1}, C_{2}, \cdots\right)$ of cycles in $G$ such that $I\left(C_{i}\right)$ is defined for all $i \geq 1$, and the following properties are satisfied:

(1) $I\left(C_{i}\right) \subset I\left(C_{i+1}\right)$ for all $i \geq 1$,

(2) $\bigcup_{i=1}^{\infty} I\left(C_{i}\right)=G$, and

(3) $C_{i} \cap C_{j}=\emptyset$ for all $i \neq j$.

Radial nets are first introduced in ([1], p. 165) along with ladder nets. If $G$ is an infinite plane graph with a radial net $N=\left(C_{1}, C_{2}, \ldots\right)$, then for any cycle $C$ in $G$, $C \subset I_{G}\left(C_{i}\right)$ for all sufficiently large $i$ (by (1) and (2) in (1.2)), and so, $I_{G}(C)$ is defined. For the same reason, if $G$ is an infinite plane graph with a radial net, then every vertex of $G$ has finite degree (that is, $G$ is locally finite).

Now we can precisely state the main result of this paper which establishes (1.1) for graphs with radial nets.

(1.3) Theorem. Let $G$ be a 4-connected plane graph which admits a radial net, let $C$ be a facial cycle of $G$, and let $e$ be an edge of $C$. Then $G$ has a spanning 2-way infinite path through $e$.

The rest of this paper is organized as follows. In the remainder of this section we introduce notation and terminology necessary for stating and proving results. In 
Section 2, we study "Tutte paths" in planar graphs. Tutte paths will be defined later, but we remark here that in 4-connected graphs Tutte paths become spanning paths. The objective of Section 2 is to show that one can construct a graph $G^{\prime}$ from a graph $G$ in a special way such that a Tutte path in $G^{\prime}$ can be extended to a Tutte path in $G$. This construction is used in Section 3 to find an infinite sequence of finite "forward" Tutte paths. We then use the "forward" property to show that these finite paths "converge" to a 2-way infinite Tutte path. Because the graph $G$ in (1.3) is 4-connected, a 2-way infinite Tutte path in $G$ is in fact a spanning path.

We use $\emptyset$ to denote both the empty set and the empty graph. Let $G$ be a graph and let $X \subset E(G)$. Then $G-X$ denotes the subgraph of $G$ with $V(G-X)=V(G)$ and $E(G-X)=E(G)-X$. The subgraph of $G$ induced by $X$ is the graph whose edge set is $X$ and whose vertex set consists of the vertices of $G$ incident with edges in $X$. Let $u_{i}, v_{i} \in V(G)$ with $u_{i} \neq v_{i}$, where $i \in I$ for some $I \subset\{1,2, \cdots\}$; then $G+\left\{u_{i} v_{i}: i \in I\right\}$ denotes the graph with vertex set $V(G)$ and edge set $E(G) \cup\left\{u_{i} v_{i}: i \in I\right\}$. For $x, y \in V(G)$, we use $G+x y$ instead of $G+\{x y\}$.

Let $P$ be a path and let $x, y$ be distinct vertices of $P$; then we use $x P y$ to denote the finite subpath of $P$ between $x$ and $y$. For a cycle $C$ in a plane graph and for distinct vertices $x, y$ of $C$, we use $x C y$ to denote the subpath of $C$ from $x$ to $y$ in clockwise order. If $G$ is a finite 2-connected plane graph, then the boundary of each face of $G$ is a cycle, and the cycle of $G$ bounding its infinite face is called the outer cycle of $G$.

For convenience, we use $A:=B$ to rename $B$ as $A$, or to define $A$ as $B$.

\section{Tutte paths}

The aim of this section is to introduce the concept of a Tutte path and to prove a technical result on Tutte paths. This result will be used in Section 3 to find finite "forward" Tutte paths which are then used to produce a 2-way infinite Tutte path.

(2.1) Definition. Let $G$ be a graph and let $P$ be a path in $G$. A $P$-bridge of $G$ is a subgraph of $G$ which either (1) is induced by a single edge in $E(G)-E(P)$ with both incident vertices in $V(P)$ or $(2)$ is induced by the edges contained in a component of $G-V(P)$ and the edges from this component to $P$. If $B$ is a $P$-bridge of $G$, then the vertices in $V(P) \cap V(B)$ are called the attachments of $B$ on $P$. We say that $P$ is a Tutte path in $G$ if every $P$-bridge of $G$ is finite and has at most three attachments. For any 
given subgraph $C$ in $G$, we say that $P$ is a $C$-Tutte path in $G$ if $P$ is a Tutte path in $G$ and every $P$-bridge of $G$ containing an edge of $C$ has at most two attachments.

Notice that if $G$ is a 4-connected planar graph and $P$ is a Tutte path in $G$ with $|V(P)| \geq 4$ then $P$ is a spanning path in $G$. Hence, Tutte paths have played important roles in finding Hamilton paths in finite graphs. We shall use Tutte paths to prove (1.3). For our purpose, we need two known results on Tutte paths in finite graphs. The first is due to Thomassen ([6], Main Theorem). In [6], a $P$-bridge is called a " $P$-component".

(2.2) Lemma. Let $G$ be a finite 2-connected plane graph with a facial cycle $C$. Assume that $u \in V(C), e \in E(C)$, and $v \in V(G)-\{u\}$. Then $G$ contains a $C$-Tutte path $P$ from $u$ to $v$ and through $e$.

Lemma (2.2) implies that in a finite 4-connected planar graph there is a spanning path between any two given vertices. The next result is due to Thomas and $\mathrm{Yu}([4]$, Lemma (2.6)). In [4], a $C$-Tutte path is called an " $E(C)$-snake".

(2.3) Lemma. Let $G$ be a finite 2-connected plane graph with a facial cycle $C$. Let $u, v \in V(C)$ be distinct, let $e, f \in E(C)$, and assume that $u, v, e, f$ occur on $C$ in this clockwise order. Then $G$ contains a $v C u$-Tutte path $P$ from $u$ to $v$ and through both $e$ and $f$.

It is easy to see that the edges $e$ and $f$ in the above lemmas can be replaced with vertices. Hence, when these lemmas are applied, we allow $e$ or $f$ or both to be vertices.

Now let us turn our attention to the main result of this section. Suppose we are given an infinite plane graph $G$, a facial cycle $C$ of $G$, and an edge $e$ of $C$. We wish to construct an infinite plane graph $G^{\prime}$, a facial cycle $C^{\prime}$ of $G^{\prime}$, and an edge $e^{\prime}$ of $C^{\prime}$ such that if $G^{\prime}$ has a $C^{\prime}$-Tutte path through $e^{\prime}$ then $G$ has a $C$-Tutte path through $e$. Intuitively, $G^{\prime}$ is obtained from an infinite block $H$ of $G-V(C)$ by adding a vertex $v^{\prime}$ of $C$ and some edges from $v^{\prime}$ to $H$. Although the statement of this result is a bit technical, it becomes natural after one reads the construction part in the beginning of the proof. (For example, conclusions (1)-(4) are obvious consequences of the construction.) Also, this statement allows us to avoid repeating the lengthy description of the construction process. We refer the readers to Figure 1 and Figure 2 for illustrations. For ease of arguments, we also add a connectivity condition. 
Let $G$ be a plane graph and let $C$ be a facial cycle of $G$. We say that $G$ is $(4, C)$ connected if $G$ is 2-connected and, for any $k$-cut $X$ of $G$ with $k \leq 3$, every component of $G-X$ contains a vertex of $C$. Clearly, if $G$ is 4 -connected then $G$ is $(4, C)$-connected.

(2.4) Theorem. Let $G$ be an infinite 2-connected plane graph, let $C$ be a facial cycle of $G$, and let $u v$ be an edge of $C$. Assume that $G$ is $(4, C)$-connected and assume that there is a cycle $C^{*}$ in $G$ such that $C \cap C^{*}=\emptyset, I_{G}\left(C^{*}\right)$ is defined, and $C \subset I_{G}\left(C^{*}\right)$. Then there exist an infinite plane graph $G^{\prime}$, a facial cycle $C^{\prime}$ of $G^{\prime}$, and a path $u^{\prime} v^{\prime} w^{\prime}$ in $C^{\prime}$ such that

(1) $G^{\prime}$ is $\left(4, C^{\prime}\right)$-connected and $G^{\prime}-v^{\prime}$ is 2-connected,

(2) $G^{\prime}-\left\{u^{\prime} v^{\prime}, v^{\prime} w^{\prime}\right\} \subset G$, and no edge of $G$ joins a vertex of $G^{\prime}-V\left(C^{\prime}\right)$ to a vertex of $G-V\left(G^{\prime}\right)$,

(3) $\left(G+\left\{u^{\prime} v^{\prime}, v^{\prime} w^{\prime}\right\}\right)-\left(V\left(G^{\prime}\right)-V\left(C^{\prime}\right)\right)$ is finite and has a plane representation with $C$ and $C^{\prime}$ as facial cycles,

(4) $v^{\prime} \neq v$ and $\left(C^{\prime}-v^{\prime}\right) \cap C=\emptyset$, and

(5) for any subgraph $X$ of $G^{\prime}$ with $C^{\prime} \subset X$, and for any $C^{\prime}$-Tutte path $P^{\prime}$ in $X$ through $u^{\prime} v^{\prime}$ and $w^{\prime}$, there is a $C$-Tutte path $P$ in $G-\left(V\left(G^{\prime}\right)-V(X)\right)$ through uv such that $P^{\prime}-v^{\prime} \subset P$ and, for any $z \in V(P)-V\left(P^{\prime}\right)$, either $z \notin V(X)$ or $z \in V(Z)$ for some $P^{\prime}$-bridge $Z$ of $X$ containing an edge of $C^{\prime}$.

Proof: The first half of the proof is devoted to the construction of $G^{\prime}, C^{\prime}, u^{\prime}, v^{\prime}, w^{\prime}$ satisfying (1)-(4), and the second half of the proof deals with (5). For convenience, we draw $I_{G}\left(C^{*}\right)$ in the plane so that $C^{*}$ is the outer cycle of $I_{G}\left(C^{*}\right)$ and $C$ is a facial cycle of $I_{G}\left(C^{*}\right)$. Hence, we can speak of the clockwise order of cycles in $I_{G}\left(C^{*}\right)$. See Figure 1.

Let $H$ be the block of $G-V(C)$ containing $C^{*}$. Then $I_{G}\left(C^{*}\right) \cap H$ is a finite 2connected plane graph. So let $D$ be the cycle bounding the face of $I_{G}\left(C^{*}\right) \cap H$ containing $C$. Note that $D$ is a also a facial cycle of $H, I_{G}(D)$ is defined, and $C \subset I_{G}(D)$. See Figure 1. Since $H$ is a block of $G-V(C)$, any $(H \cup C)$-bridge of $G$ has at most one attachment on $H$. By planarity, all attachments on $H$ of $(H \cup C)$-bridges of $G$ are contained in $V(D)$.

So let $w_{1}, \cdots, w_{b}$ be the attachments on $D$ of $(H \cup C)$-bridges of $G$, and assume that $w_{1}, \ldots, w_{b}$ occur on $D$ in this clockwise order. For each $j \in\{1, \ldots, b\}$, let $p_{j}, q_{j} \in V(C)$ 



Figure 1: Plane representations of $I_{G}\left(C^{*}\right)$ and $I_{G^{\prime}}\left(C^{*}\right)$

such that (a) $\left\{p_{j}, w_{j}\right\}$ is contained in an $(H \cup C)$-bridge of $G$ and $\left\{q_{j}, w_{j}\right\}$ is contained in an $(H \cup C)$-bridge of $G,(\mathrm{~b})$ any $(H \cup C)$-bridge of $G$ containing some $w_{i}$ with $w_{i} \neq w_{j}$ contains no vertex of $p_{j} C q_{j}-\left\{p_{j}, q_{j}\right\}$, and (c) subject to (a) and (b), $p_{j} C q_{j}$ is maximal. See Figure 1. Since $G$ is a plane graph and because $G$ is $(4, C)$-connected, $p_{j}$ and $q_{j}$ are well defined, and $p_{1}, q_{1}, p_{2}, q_{2}, \ldots, p_{b}, q_{b}$ occur on $C$ in the clockwise order listed. Let $J_{j}$ denote the union of $p_{j} C p_{j+1}$ and those $(H \cup C)$-bridges of $G$ whose attachments are all contained in $V\left(p_{j} C p_{j+1}\right) \cup\left\{w_{j}\right\}$.

Without loss of generality, assume that $u v \in E\left(J_{k}\right)$ for some positive integer $k$, and $p_{k}, u, v, p_{k+1}$ occur on $C$ in this clockwise order. Choose $w_{l}$ such that $p_{l} \neq p_{k}$ and, subject to this, $w_{l} D w_{k}$ is minimal. In Figure $1, J_{k}$ and $J_{l}$ are marked with dotted curves.

We claim that $p_{k} \neq p_{k+1}$ (where $p_{b+1}:=p_{1}$ ). Suppose on the contrary that $p_{k}=p_{k+1}$. Since $u v \in E\left(J_{k}\right), C \subset J_{k}$. Hence $\left\{p_{k}, w_{k}\right\}$ is a 2-cut of $G$ and $G-\left\{p_{k}, w_{k}\right\}$ has a component containing no vertex of $C$. This contradicts the assumption that $G$ is $(4, C)$-connected.

Note that if $w_{j} \in V\left(w_{l} D w_{k}\right)-\left\{w_{l}, w_{k}\right\}$, then by the choice of $w_{l}, p_{j}=q_{j}=p_{k}$ (in particular, $\left.p_{l+1}=p_{k}\right)$. Hence, since $G$ is $(4, C)$-connected, $J_{j}$ is a subgraph of $G$ induced by the edge $p_{k} w_{j}$ for each $w_{j} \in V\left(w_{l} D w_{k}\right)-\left\{w_{l}, w_{k}\right\}$.

Let $u^{\prime}=w_{l}, v^{\prime}=p_{k}$, and $w^{\prime}=w_{k}$. Let $G^{\prime}$ denote the graph obtained from $H$ by adding $v^{\prime}$ and edges $v^{\prime} w_{j}$ for all $w_{j} \in V\left(w_{l} D w_{k}\right)$, and let $C^{\prime}$ denote the cycle obtained from $w^{\prime} D u^{\prime}$ by adding $v^{\prime}$ and edges $u^{\prime} v^{\prime}$ and $v^{\prime} w^{\prime}$. This completes the description of $G^{\prime}, C^{\prime}, u^{\prime}, v^{\prime}$ and $w^{\prime}$. See Figure 1 for a plane representation of $I_{G^{\prime}}\left(C^{*}\right)$. This finishes 
the description of the construction.

Clearly by the above construction, $G^{\prime}-v^{\prime}=H$ is 2 -connected. To prove (1), we need to show that $G^{\prime}$ is $\left(4, C^{\prime}\right)$-connected. Suppose for a contradiction that $G^{\prime}$ has a $k$-cut $S$ with $k \leq 3$ and $G^{\prime}-S$ has a component $K$ not containing any vertex of $C^{\prime}$. Then by planarity, $S$ is a $k$-cut of $G$ and $K$ is a component of $G-S$ not containing any vertex of $C$, contradicting the assumption that $G$ is $(4, C)$-connected. Thus (1) holds.

From the above construction, we see that $G^{\prime}$ is obtained from $H \subset G$ by adding $v^{\prime}=p_{k}$ and by adding edges $v^{\prime} w_{j}$ for all $w_{j} \in V\left(w_{l} D w_{k}\right)$. We already noted above that $J_{j}$ is a subgraph of $G$ induced by $v^{\prime} w_{j}$ for all $w_{j} \in V\left(w_{l} D w_{k}\right)-\left\{w_{k}, w_{l}\right\}$. So $G^{\prime}-\left\{u^{\prime} v^{\prime}, v^{\prime} w^{\prime}\right\} \subset G$. By planarity, no edge of $G$ joins a vertex of $G^{\prime}-V\left(C^{\prime}\right)=$ $H-V\left(w_{k} D w_{l}\right)$ to a vertex of $G-V\left(G^{\prime}\right)=G-\left(V(H) \cup\left\{p_{k}\right\}\right)$. So we have (2).

Because $G-\left(V\left(G^{\prime}\right)-V\left(C^{\prime}\right)\right)$ is contained in $I_{G}\left(C^{*}\right)$, we see that $G-\left(V\left(G^{\prime}\right)-V\left(C^{\prime}\right)\right)$ is a finite graph. Moreover, it is easy to see that $\left(G+\left\{u^{\prime} v^{\prime}, v^{\prime} w^{\prime}\right\}\right)-\left(V\left(G^{\prime}\right)-V\left(C^{\prime}\right)\right)$ has a plane representation such that $C$ and $C^{\prime}$ are its facial cycles. So we have (3).

By the above construction, $\left(C^{\prime}-v^{\prime}\right) \cap C=\emptyset$. Since $p_{k}, u, v, p_{k+1}$ occur on $C$ in this clockwise order and $p_{k} \neq p_{k+1}$, we have $v^{\prime} \neq v$. So (4) holds.

Now let us turn our attention to (5). Let $X$ be a subgraph of $G^{\prime}$ with $C^{\prime} \subset X$, and let $P^{\prime}$ be a $C^{\prime}$-Tutte path in $X$ through $u^{\prime} v^{\prime}$ and $w^{\prime}$. We wish to find a $C$-Tutte path $P$ in $G-\left(V\left(G^{\prime}\right)-V(X)\right)$ through $u v$ such that $P^{\prime}-v^{\prime} \subset P$ and, for any $z \in V(P)-V\left(P^{\prime}\right)$, either $z \notin V(X)$ or $z \in V(Z)$ for some $P^{\prime}$-bridge $Z$ of $X$ containing an edge of $C^{\prime}$. Note that $G-\left(V\left(G^{\prime}\right)-X\right)$ is the union of $C, X-\left\{u^{\prime} v^{\prime}, v^{\prime} w^{\prime}\right\}$, and those $(H \cup C)$-bridges of $G$ whose attachments are all contained in $V\left(w_{k} D w_{l}\right) \cup V(C)$. See Figure 2.

Let $W$ denote the set of attachments on $w_{k} D w_{l}$ of $(H \cup C)$-bridges of $G$. (Hence, $W \subset\left\{w_{1}, \cdots, w_{b}\right\}$.) We define an equivalence relation on $W$ as follows. For $w, w^{\prime} \in W$, we say $w \sim w^{\prime}$ if $w=w^{\prime}$ or there is a $P^{\prime}$-bridge $B$ of $X$ such that $\left\{w, w^{\prime}\right\} \subset V(B)-V\left(P^{\prime}\right)$. Let $W_{1}, W_{2}, \cdots, W_{m}$ denote the equivalence classes of $W$ with respect to $\sim$. Thus, $\left|W_{i}\right|=1$ if $W_{i} \subset V\left(P^{\prime}\right)$. If $W_{i} \not \subset V\left(P^{\prime}\right)$, then $W_{i}=\left(V\left(B_{i}\right)-V\left(P^{\prime}\right)\right) \cap W$ for some $P^{\prime}$-bridge $B_{i}$ of $X$. Without loss of generality, let $W_{1}=\left\{w_{k}\right\}$ and $W_{m}=\left\{w_{l}\right\}$ (because $\left.w_{k}, w_{l} \in V\left(P^{\prime}\right)\right)$, and assume that $W_{1}, W_{2}, \cdots, W_{m}$ occur on $D$ in this clockwise order.

For each $i \in\{1, \cdots, m\}$, let $s_{i}, t_{i} \in V(C)$ such that (a) $p_{k}, s_{i}, t_{i}, p_{l}$ occur on $C$ in this clockwise order, (b) there is some $w_{s} \in W_{i}$ such that $\left\{s_{i}, w_{s}\right\}$ is contained in an $(H \cup C)$-bridge of $G$, and there is some $w_{t} \in W_{i}$ such that $\left\{t_{i}, w_{t}\right\}$ is contained in an 




$G-\left(V\left(G^{\prime}\right)-X\right)$

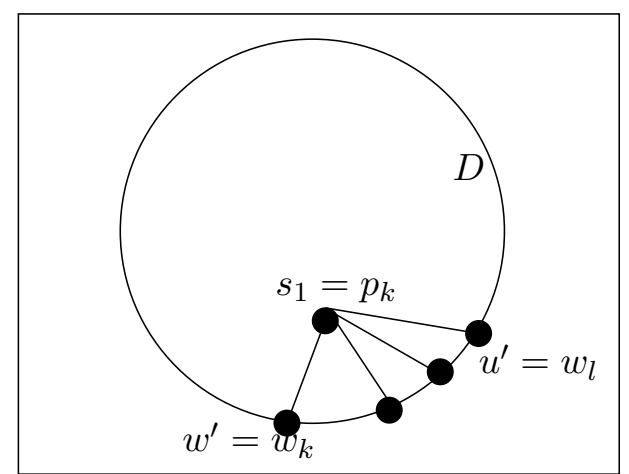

$X$

Figure 2: $X$ and $G-\left(V\left(G^{\prime}\right)-X\right)$

$(H \cup C)$-bridge of $G$, and (c) subject to (a) and (b), $s_{i} C t_{i}$ is maximal. Then $s_{1}=p_{k}=v^{\prime}$, $s_{2}=p_{k+1}$, and $s_{m}=p_{l}$. See Figure 2. Moreover, $s_{1}, t_{1}, s_{2}, t_{2}, \ldots, s_{m}, t_{m}$ occur on $C$ in the clockwise order listed.

For each $i \in\{2, \cdots, m-1\}$, let $T_{i}$ denote the union of $t_{i} C s_{i+1}$ and those $(H \cup C)$ bridges of $G$ whose attachments are all contained in $V\left(t_{i} C s_{i+1}\right)$. See Figure 3 (without the dashed edge). Note that if $\left|V\left(T_{i}\right)\right| \geq 3$ then $t_{i} \neq s_{i+1}$ and $\left\{t_{i}, s_{i+1}\right\}$ is a 2-cut of $G$.

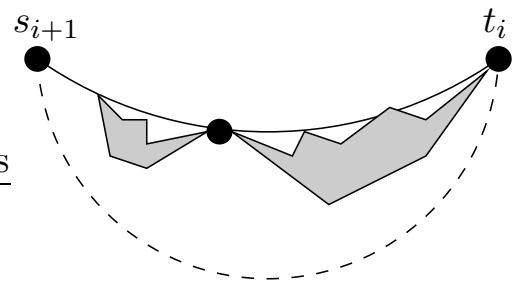

Figure $3: T_{i}+t_{i} s_{i+1}$

For each $i \in\{2, \cdots, m-1\}$, let $B_{i}=W_{i}$ if $W_{i} \subset V\left(P^{\prime}\right)$ (in this case, $\left|W_{i}\right|=1$ ); and otherwise, let $B_{i}$ denote the $P^{\prime}$-bridge of $X$ such that $W_{i}=\left(V\left(B_{i}\right)-V\left(P^{\prime}\right)\right) \cap W$. Let $U_{i}$ denote the union of $s_{i} C t_{i}, B_{i}$, and those $(H \cup C)$-bridges of $G$ whose attachments are all contained in $V\left(s_{i} C t_{i}\right) \cup W_{i}$. Then $\left|V\left(U_{i}\right) \cap V\left(P^{\prime}\right)\right|=\left|V\left(B_{i}\right) \cap V\left(P^{\prime}\right)\right| \leq 2$. See Figure 4 (without the dashed edges).

Note that $U_{i} \cap C=s_{i} C t_{i}$ and $T_{i} \cap C=t_{i} C s_{i+1}$. Also note that $U_{i}-\left(V(C) \cup V\left(P^{\prime}\right)\right)$, $T_{i}-V(C), i=2, \ldots, m-1$, are pair-wise disjoint. 


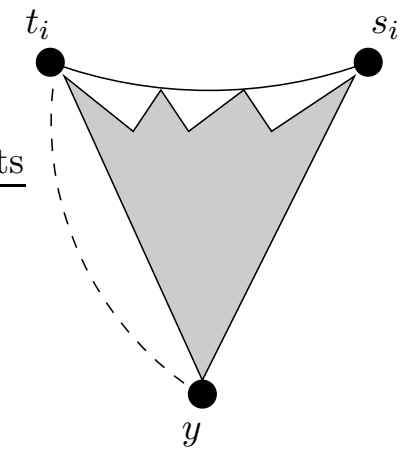

$W_{i}=\{y\} \subset V\left(P^{\prime}\right)$

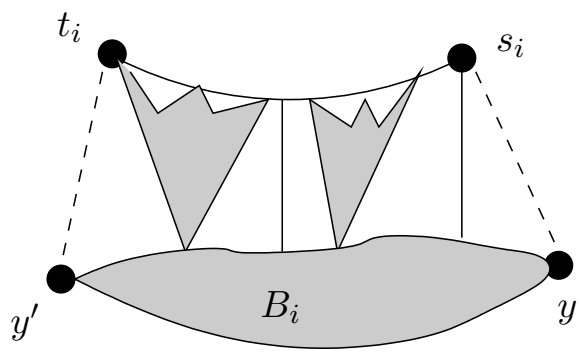

$W_{i} \not \subset V\left(P^{\prime}\right)$

Figure 4: $U_{i}+y t_{i}$ and $U_{i}+\left\{s_{i} y, y^{\prime} t_{i}\right\}$

We shall construct the desired path $P$ in (5) by finding the following paths: a path $Q_{1}$ in $J_{k}$ through $u v$ which is from $w^{\prime}=w_{k}$ to $s_{2}=p_{k+1}$ (when $w^{\prime} v^{\prime} \in E\left(P^{\prime}\right)$ ) or from $v^{\prime}=p_{k}$ to $s_{2}=p_{k+1}\left(\right.$ when $\left.w^{\prime} v^{\prime} \notin E\left(P^{\prime}\right)\right)$, a path $Q_{m}$ in $J_{l}-v^{\prime}$ from $u^{\prime}=w_{l}$ to $s_{m}=p_{l}$, a path $Q_{i}$ in $U_{i}-V\left(P^{\prime}\right)$ from $s_{i}$ to $t_{i}$ for each $i \in\{2, \ldots, m-1\}$, and a path $R_{i}$ in $T_{i}$ from $t_{i}$ to $s_{i+1}$ for each $i \in\{2, \ldots, m-1\}$. First, we prove the following statement.

(I) If $w^{\prime} v^{\prime} \in E\left(P^{\prime}\right)$ then there is a $\left(J_{k} \cap C\right)$-Tutte path $Q_{1}$ from $w^{\prime}$ to $s_{2}$ and through both $v^{\prime}$ and $u v$; if $u^{\prime} v^{\prime} \notin E\left(P^{\prime}\right)$ then there is a path $Q_{1}$ in $J_{k}-w^{\prime}$ from $v^{\prime}$ to $s_{2}$ such that every $\left(Q_{1} \cup\left\{w^{\prime}\right\}\right)$-bridge of $J_{k}$ has at most three attachments, and every $\left(Q_{1} \cup\left\{w^{\prime}\right\}\right)$ bridge of $J_{k}$ containing an edge of $J_{k} \cap C$ has just two attachments.

Since $G$ is 2-connected, any cut vertex of $J_{k}$ must separate $v^{\prime}$ from $s_{2}$ or separate $w^{\prime}$ from $v^{\prime} C s_{2}$. Since $\left\{w^{\prime}, v^{\prime}\right\}$ is contained in an $(H \cup C)$-bridge of $G, J_{k}^{*}:=J_{k}+w^{\prime} s_{2}$ is 2-connected. We may add the edge $w^{\prime} s_{2}$ so that $v^{\prime} C s_{2}$ and $w^{\prime} s_{2}$ are contained in the outer cycle $C_{k}$ of $J_{k}^{*}$ and $w^{\prime}, s_{2}, v, u, v^{\prime}$ occur on $C_{k}$ in this clockwise order. See Figure 5. Note that $J_{k} \cap C=s_{2} C_{k} v^{\prime}=v^{\prime} C s_{2}$.

If $w^{\prime} v^{\prime} \in E\left(P^{\prime}\right)$, then we apply (2.3) (with $J_{k}^{*}, C_{k}, w^{\prime}, s_{2}, u v, v^{\prime}$ as $G, C, u, v, e, f$ in $(2.3)$, respectively) to find an $s_{2} C_{k} w^{\prime}$-Tutte path $Q_{1}$ in $J_{k}^{*}$ from $w^{\prime}$ to $s_{2}$ and through $v^{\prime}$ and $u v$. Note that $w^{\prime} s_{2} \notin E\left(Q_{1}\right)$. Hence $Q_{1}$ gives the desired path for (I).

Now assume that $w^{\prime} v^{\prime} \notin E\left(P^{\prime}\right)$. We apply (2.3) (with $J_{k}^{*}, C_{k}, v^{\prime}, w^{\prime}, w^{\prime} s_{2}, u v$ as $G, C, u, v, e, f$ in (2.3), respectively) to find a $w^{\prime} C_{k} v^{\prime}$-Tutte path $Q_{1}^{\prime}$ in $J_{k}^{*}$ from $v^{\prime}$ to $w^{\prime}$ and through both $u v$ and $w^{\prime} s_{2}$; and let $Q_{1}:=Q_{1}^{\prime}-w^{\prime}$. Note that $Q_{1}$ is a path in $J_{k}-w^{\prime}$ from $v^{\prime}$ to $s_{2}$ and through $u v$. Also note that every $\left(Q_{1} \cup\left\{w^{\prime}\right\}\right)$-bridge of $J_{k}$ is a $Q_{1}^{\prime}$-bridge of $J_{k}^{*}$, except possibly the subgraph of $G$ induced by the edge $w^{\prime} s_{2}$. So $Q_{1}$ 
gives the desired path for (I).



Figure 5: $J_{k}^{*}$

(II) We claim that $J_{l}-v^{\prime}$ has a path $Q_{m}$ from $u^{\prime}=w_{l}$ to $s_{m}$ such that every $\left(Q_{m} \cup\left\{v^{\prime}\right\}\right)$-bridge of $J_{l}$ has at most three attachments, and every $\left(Q_{m} \cup\left\{v^{\prime}\right\}\right)$-bridge of $J_{l}$ containing an edge of $J_{l} \cap C$ has just two attachments.

Let $J_{l}^{*}:=J_{l}+u^{\prime} v^{\prime}$. By the same argument as in (I) for $J_{k}^{*}$, we can show that $J_{l}^{*}$ is 2-connected. We may add the edge $u^{\prime} v^{\prime}$ so that $s_{m} C v^{\prime}$ and $u^{\prime} v^{\prime}$ are contained in the outer cycle $C_{l}$ of $J_{l}^{*}$, and $u^{\prime}, v^{\prime}, s_{m}$ occur on $C_{l}$ in this clockwise order. See Figure 6 . Note that $J_{l} \cap C=v^{\prime} C_{l} s_{m}=s_{m} C v^{\prime}$.

We apply (2.2) (with $J_{l}^{*}, C_{l}, v^{\prime}, s_{m}, u^{\prime} v^{\prime}$ as $G, C, u, v, e$ in (2.2), respectively) to find a $C_{l}$-Tutte path $Q_{m}^{\prime}$ in $J_{l}^{*}$ from $v^{\prime}$ to $s_{m}$ through $u^{\prime} v^{\prime}$; and let $Q_{m}:=Q_{m}^{\prime}-v^{\prime}$. Note that $Q_{m}$ is a path in $J_{l}-v^{\prime}$ from $u^{\prime}=w_{l}$ to $s_{m}$. Also note that every $\left(Q_{m} \cup\left\{v^{\prime}\right\}\right)$-bridge of $J_{l}$ is a $Q_{m}^{\prime}$-bridge of $J_{l}^{*}$, except possibly the subgraph of $G$ induced by the edge $u^{\prime} v^{\prime}$. Hence $Q_{m}$ gives the desired path for (II).

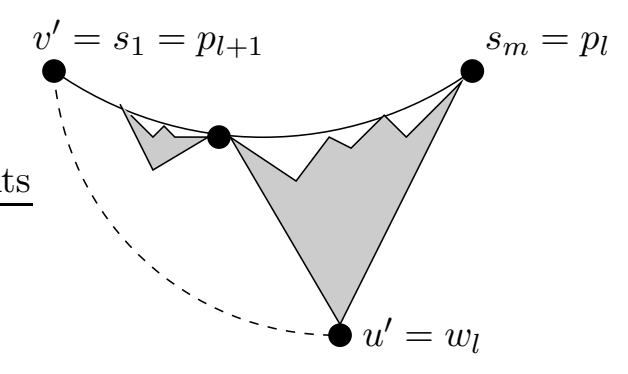

Figure 6: $J_{l}^{*}$

(III) For each $i \in\{2, \ldots, m-1\}$, there is a $t_{i} C s_{i+1}$-Tutte path $R_{i}$ in $T_{i}$ from $t_{i}$ to $s_{i+1}$.

If $\left|V\left(t_{i} C s_{i+1}\right)\right| \leq 2$, then $R_{i}:=t_{i} C s_{i+1}$ gives the desired path. Now assume that $\left|V\left(t_{i} C s_{i+1}\right)\right| \geq 3$. Note that $T_{i} \cap D=\emptyset$, and every cut vertex of $T_{i}$ must separate $t_{i}$ from 
$s_{i+1}$ (since $G$ is 2 -connected). Hence, $T_{i}^{\prime}:=T_{i}+t_{i} s_{i+1}$ is 2 -connected. We may add the edge $t_{i} s_{i+1}$ so that $C_{i}:=t_{i} C s_{i+1}+t_{i} s_{i+1}$ is the outer cycle of $T_{i}^{\prime}$. See Figure 3. By (2.2) (with $T_{i}^{\prime}, C_{i}, t_{i}, s_{i+1}$ as $G, C, u, v$ in $(2.2)$, respectively), we find a $C_{i}$-Tutte path $R_{i}$ in $T_{i}^{\prime}$ from $t_{i}$ to $s_{i+1}$ and through an edge of $t_{i} C s_{i+1}$. It is easy to see that $t_{i} s_{i+1} \notin E\left(R_{i}\right)$, and so, $R_{i}$ gives the desired path in $T_{i}$.

(IV) We claim that, for each $i \in\{2, \cdots, m-1\}, U_{i}-V\left(P^{\prime}\right)$ contains a path $Q_{i}$ from $s_{i}$ to $t_{i}$ such that every $\left(Q_{i} \cup\left(U_{i} \cap P^{\prime}\right)\right)$-bridge of $U_{i}$ has at most three attachments, and any $\left(Q_{i} \cup\left(U_{i} \cap P^{\prime}\right)\right)$-bridge of $U_{i}$ containing an edge of $U_{i} \cap C$ has just two attachments.

Recall that $U_{i} \cap C=s_{i} C t_{i}$ and $\left|V\left(U_{i}\right) \cap V\left(P^{\prime}\right)\right| \leq 2$.

If $s_{i}=t_{i}$, then let $Q_{i}:=s_{i} C t_{i}$. In this case, $\left|V\left(U_{i}\right) \cap V(C)\right|=1$. Hence, since $\left|V\left(U_{i}\right) \cap V\left(P^{\prime}\right)\right| \leq 2, Q_{i}$ gives the desired path. So we may assume that $s_{i} \neq t_{i}$. We consider two cases.

First, assume $W_{i} \subset V\left(P^{\prime}\right)$. Then $\left|W_{i}\right|=1$. Let $y$ be the only vertex in $W_{i}$. Then $U_{i} \cap P^{\prime}$ consists of the vertex $y$. See Figure 4 . Since $G$ is 2 -connected, any cut vertex of $U_{i}$ must separate $y$ from $s_{i} C t_{i}$. Hence, $U_{i}^{\prime}:=U_{i}+t_{i} y$ is 2-connected. We add the edge $t_{i} y$ so that $s_{i} C t_{i}$ and $t_{i} y$ are contained in the outer cycle $C_{i}$ of $U_{i}^{\prime}$. By (2.2) (with $U_{i}^{\prime}, C_{i}, s_{i}, y, t_{i} y$ as $G, C, u, v, e$ in $(2.2)$, respectively), we find a $C_{i}$-Tutte path $Q_{i}^{\prime}$ in $U_{i}^{\prime}$ from $s_{i}$ to $y$ and through $t_{i} y$; and let $Q_{i}:=Q_{i}^{\prime}-y$. Then $Q_{i} \subset U_{i}-V\left(P^{\prime}\right)$. Since $U_{i} \cap C=s_{i} C t_{i}$ and because $U_{i} \cap P^{\prime}$ consists of $y$ only, it is easy to check that $Q_{i}$ is the desired path.

Now assume that $W_{i} \not \subset V\left(P^{\prime}\right)$. Then $W_{i}=\left(V\left(B_{i}\right)-V\left(P^{\prime}\right)\right) \cap W$ for some $P^{\prime}$-bridge $B_{i}$ of $X$ containing an edge of $C^{\prime}$. Hence, $B_{i}$ has two attachments on $P^{\prime}$, say $y$ and $y^{\prime}$. Note that $U_{i} \cap P^{\prime}$ consists of $y$ and $y^{\prime}$. See Figure 4. Without loss of generality, we may assume that $w^{\prime}, y, y^{\prime}, u^{\prime}$ occur on $C^{\prime}$ in this clockwise order. Since $G$ is 2-connected, any cut vertex of $U_{i}$ either separates $s_{i} C t_{i}$ from $B_{i}$ or separates $y$ from $y^{\prime}$. Hence, $U_{i}^{\prime}:=U_{i}+\left\{s_{i} y, y^{\prime} t_{i}\right\}$ is 2-connected. We add the edges $s_{i} y$ and $y^{\prime} t_{i}$ so that $s_{i} C t_{i}, s_{i} y$, $y^{\prime} t_{i}$ are contained in the outer cycle $C_{i}$ of $U_{i}^{\prime}$. By applying (2.3) (with $U_{i}^{\prime}, C_{i}, y, y^{\prime}, y^{\prime} t_{i}, s_{i} y$ as $G, C, u, v, e, f$ in (2.3), respectively), we find a $y^{\prime} C_{i} y$-Tutte path $Q_{i}^{\prime}$ in $U_{i}^{\prime}$ from $y$ to $y^{\prime}$ and through both $s_{i} y$ and $y^{\prime} t_{i}$. Let $Q_{i}:=Q_{i}^{\prime}-\left\{y, y^{\prime}\right\}$. Then $Q_{i} \subset U_{i}-V\left(P^{\prime}\right)$. Since $U_{i} \cap C=s_{i} C t_{i}$ and because $U_{i} \cap P^{\prime}$ consists of $y$ and $y^{\prime}$, it is easy to check that $Q_{i}$ gives the desired path.

(V) Finally, let us construct the desired path $P$. If $w^{\prime} v^{\prime} \in E\left(P^{\prime}\right)$ then $Q_{1}$ is a path in $J_{k}$ from $w^{\prime}$ to $s_{2}$ and through both $v^{\prime}$ and $u v$, and in this case we let $P:=$ 
$\left(\bigcup_{i=1}^{m} Q_{i}\right) \cup\left(\bigcup_{i=2}^{m-1} R_{i}\right) \cup\left(P^{\prime}-v^{\prime}\right)$. If $w^{\prime} v^{\prime} \notin E\left(P^{\prime}\right)$ then $Q_{1}$ is a path in $J_{k}-w^{\prime}$ from $v^{\prime}$ to $s_{2}$ and through $u v$, and in this case we let $P:=\left(\bigcup_{i=1}^{m} Q_{i}\right) \cup\left(\bigcup_{i=2}^{m-1} R_{i}\right) \cup\left(P^{\prime}-u^{\prime} v^{\prime}\right)$. Note that $u^{\prime} v^{\prime} \notin E(P)$, and if $v^{\prime} w^{\prime} \notin E(G)$ then $v^{\prime} w^{\prime} \notin E(P)$. It is clear that $P$ is a path in $G-\left(V\left(G^{\prime}\right)-V(X)\right)$ through $u v$.

To prove that $P$ is a $C$-Tutte path in $G-\left(V\left(G^{\prime}\right)-V(X)\right)$, let $B$ be a $P$-bridge of $G-\left(V\left(G^{\prime}\right)-V(X)\right)$. It is straightforward to check that one of the following holds: $B$ is induced by a single edge in $E(G)-E(P)$ with both incident vertices in $V(P)$; or $B$ is a $P^{\prime}$-bridge of $X$ such that $\left(V(B)-V\left(P^{\prime}\right)\right) \cap\left\{w_{1}, \cdots, w_{b}\right\}=\emptyset$; or $B$ is obtained from a $P^{\prime}$-bridge $B^{\prime}$ of $X$ with $\left(V\left(B^{\prime}\right)-V\left(P^{\prime}\right)\right) \cap\left\{w_{1}, \cdots, w_{b}\right\} \subset V\left(w_{l} D w_{k}\right)$ by adding $v^{\prime}$ and $v^{\prime} w_{j}$ for all $w_{j} \in V\left(B^{\prime}\right)-V\left(P^{\prime}\right)$; or $B$ is a $Q_{1}$-bridge of $J_{k}$ when $w^{\prime} v^{\prime} \in E\left(P^{\prime}\right)$; or $B$ is a $\left(Q_{1} \cup\left\{w^{\prime}\right\}\right)$-bridge of $J_{k}$ when $w^{\prime} v^{\prime} \notin E\left(P^{\prime}\right)$ (because $\left.w^{\prime} \in V\left(P^{\prime}\right)\right)$; or $B$ is a $\left(Q_{m} \cup\left\{v^{\prime}\right\}\right)$-bridge of $J_{l}$; or $B$ is a $\left(Q_{i} \cup\left(U_{i} \cap P^{\prime}\right)\right)$-bridge of $U_{i}$ for some $i \in\{2, \cdots, m-1\}$; or $B$ is a $R_{i}$-bridge of $T_{i}$ for some $i \in\{2, \cdots, m-1\}$. Hence, it is easy to see that $B$ has at most three attachments on $P$, and if $B$ contains an edge of $C$ then $B$ has just two attachments on $P$. Therefore, $P$ is a $C$-Tutte path in $G$ through $u v$.

Clearly, $P^{\prime}-v^{\prime} \subset P$. To complete the proof of (5), let $z \in V(P)-V\left(P^{\prime}\right)$. Then either $z \notin V(X)$ or $z \in V\left(B_{i}\right)-V\left(P^{\prime}\right)$ for some $i \in\{2, \ldots, m-1\}$. Recall that $Z:=B_{i}$ is a $P^{\prime}$-bridge of $X$ containing an edge of $C^{\prime}$. Thus, $P$ gives the desired path.

\section{2-Way infinite paths}

In this section, we prove (1.3). In fact, we prove a slightly stronger result. First, we prove the following result which allows us to "construct" a 2-way infinite path from a sequence of finite paths. We say that a sequence of finite paths $\left\{P_{n}\right\}$ converges to a path $P$ if for any given $u, v \in V(P), u P v=u P_{n_{k}} v$ for all sufficiently large $n_{k}$.

(3.1) Lemma. Let $G$ be an infinite locally finite graph with $e \in E(G)$. Suppose $\left\{P_{n}\right\}$ is an infinite sequence of finite paths through e such that for all $n \geq 1$, the length of each component of $P_{n+1}-e$ is strictly larger than the length of any component of $P_{n}-e$. Then $\left\{P_{n}\right\}$ has an infinite subsequence $\left\{P_{n_{k}}\right\}$ converging to a 2-way infinite path $P$ through $e$.

Proof: Let $Z^{+}=\{1,2,3, \ldots\}$ and let $e=x y$. Because $G$ is locally finite and since the length of components of $P_{n}-e$ increases with $n$, there exist $x_{1} x, y_{1} y \in E(G)$ and an infinite set $A_{1} \subset Z^{+}$such that for every $n \in A_{1}, x_{1} x y y_{1}$ is a subpath of $P_{n}$. By 
the same reason, there exist $x_{2} x_{1}, y_{2} y_{1} \in E(G)$ and an infinite set $A_{2} \subset A_{1}$ such that for every $n \in A_{2}, x_{2} x_{1} x y y_{1} y_{2}$ is a subpath of $P_{n}$. Continuing this process, we produce a 2-way infinite path $P:=\ldots x_{2} x_{1} x y y_{1} y_{2} \ldots$ and an infinite sequence of infinite sets $A_{1} \supset A_{2} \supset A_{3} \ldots$ such that for any given $k \in Z^{+}, x_{k} \ldots x_{1} x y y_{1} \ldots y_{k}$ is a subpath of $P_{n}$ for all $n \in A_{k}$.

Let $n_{k} \in A_{k}$ such that the sequence $\left\{n_{k}\right\}$ increases; this can be done because each $A_{i}$ is infinite. Therefore, $\left\{P_{n_{k}}\right\}$ is a subsequence of $\left\{P_{n}\right\}$. Let $u, v$ be two distinct vertices on $P$. Then $u, v \in V\left(x_{l} P y_{l}\right)$ for some sufficiently large $l$. Then $u P v \subset P_{n_{k}}$ for all $k \geq l$. Hence, $\left\{P_{n_{k}}\right\}$ converges to $P$.

In later proofs, we need to find a sequence of finite Tutte paths which converge to a 2-way infinite Tutte path. For this reason, we need those finite Tutte paths to be "forward".

(3.2) Definition. Let $N=\left(C_{1}, C_{2}, \cdots\right)$ be a radial net in an infinite plane graph $G$. We say that a path $P$ in $G$ is $N$-forward or $\left(C_{1}, C_{2}, \cdots\right)$-forward if, for every $i \geq 1$ and for any distinct $x, y, z \in V(P)$ with $y \in V(x P z),\{x, z\} \subset V\left(C_{i}\right)$ implies that $y \notin V\left(C_{j}\right)$ for all $j \geq i+2$.

Intuitively, " $P$ is $\left(C_{1}, C_{2}, \cdots\right)$-forward" means that if $P$ starts from $C_{1}$, then (for each $i$ ) after $P$ hits $C_{i+2}, P$ never comes back to $C_{i}$ again. Next we show how finite forward Tutte paths converge to a 2-way infinite Tutte path.

(3.3) Lemma. Let $G$ be an infinite 2-connected plane graph with a radial net $N=$ $\left(C_{1}, C_{2}, \cdots\right)$, let $C$ be a facial cycle of $G$ with $C \subset I\left(C_{1}\right)$, and let $e$ be an edge of $C$. Suppose that, for all $n \geq 1$, there exists a $C$-Tutte path $P_{n}$ in $I\left(C_{n}\right)$ between two vertices of $C_{n}$ and through $e$ such that each component of $P_{n}-e$ is an $N$-forward path in $G$. Then $\left\{P_{n}\right\}$ has a subsequence $\left\{P_{n_{k}}\right\}$ converging to a 2-way infinite $C$-Tutte path $P$ in $G$ through $e$.

Proof: Since $G$ admits a radial net, $G$ is locally finite. Since $I\left(C_{i}\right) \subset I\left(C_{i+1}\right)$ and $C_{i} \cap C_{i+1}=\emptyset$, and because $P_{n}$ is between two vertices of $C_{n}$ and through $e,\left\{P_{n}\right\}$ contains a subsequence $\left\{P_{n_{s}}\right\}$ such that the length of each component of $P_{n_{s}}-e$ increases. By (3.1), $\left\{P_{n_{s}}\right\}$ contains a subsequence $\left\{P_{n_{k}}\right\}$ converging to a 2 -way infinite path $P$ through $e$. We need to prove that $P$ is a $C$-Tutte path in $G$. First, we claim that

(1) for any given integer $l \geq 1, P_{n_{k}} \cap I\left(C_{l}\right)=P \cap I\left(C_{l}\right)$ for all sufficiently large $n_{k}$. 
Let $y_{1}, y_{2} \in V(P) \cap V\left(I\left(C_{l}\right)\right)$ with $y_{1} P y_{2}$ maximal. Then $P \cap I\left(C_{l}\right)=y_{1} P y_{2} \cap I\left(C_{l}\right)$. Since $\left\{P_{n_{k}}\right\}$ converges to $P, y_{1} P_{n_{k}} y_{2}=y_{1} P y_{2}$ for all sufficiently large $n_{k}$. Hence, $P \cap I\left(C_{l}\right)=y_{1} P y_{2} \cap I\left(C_{l}\right)=y_{1} P_{n_{k}} y_{2} \cap I\left(C_{l}\right) \subset P_{n_{k}} \cap I\left(C_{l}\right)$ for all sufficiently large $n_{k}$.

It remains to show that $P_{n_{k}} \cap I\left(C_{l}\right) \subset P \cap I\left(C_{l}\right)$ for all sufficiently large $n_{k}$. Let $x, y \in V(P) \cap V\left(C_{l+2}\right)$ such that $x$ and $y$ are contained in different components of $P-e$. Since $\left\{P_{n_{k}}\right\}$ converges to $P, x P y=x P_{n_{k}} y$ for all sufficiently large $n_{k}$.

We claim that for each sufficiently large $n_{k}$, if $z \in V\left(P_{n_{k}}\right)-V\left(x P_{n_{k}} y\right)$ then $z \notin$ $V\left(I\left(C_{l}\right)\right)$. Suppose for a contradiction that $z \in V\left(I\left(C_{l}\right)\right)$. By symmetry between $x$ and $y$, we may assume that $z$ and $y$ are contained in the same component $L$ of $P_{n_{k}}-e$. Then $z P_{n_{k}} y$ contains a vertex $z^{\prime}$ of some $C_{i}, i \leq l$. Since $e \in E\left(I\left(C_{1}\right)\right)$, there is a vertex $x^{\prime}$ in the subpath of $P_{n_{k}}$ between $e$ and $y$ such that $x^{\prime} \in V\left(C_{i}\right)$. Since $z \in V\left(P_{n_{k}}\right)-V\left(x P_{n_{k}} y\right)$, $x^{\prime} \neq z^{\prime}$ and $y \in V\left(x^{\prime} L z^{\prime}\right)$. Since $L$ is $\left(C_{1}, C_{2}, \cdots\right)$-forward and because $x^{\prime}, z^{\prime} \in V\left(C_{i}\right)$, $y \notin V\left(C_{j}\right)$ for all $j \geq i+2$, contradicting the assumption that $y \in V\left(C_{l+2}\right)$.

Thus, for all sufficiently large $n_{k}, P_{n_{k}} \cap I\left(C_{l}\right)=x P_{n_{k}} y \cap I\left(C_{l}\right)=x P y \cap I\left(C_{l}\right) \subset$ $P \cap I\left(C_{l}\right)$. This completes the proof of (1).

Now let $B$ be a $P$-bridge of $G$. We claim that

(2) $B$ is finite.

Suppose that $B$ is infinite. Since $G$ (and hence $B-V(P)$ ) is locally finite and $B-V(P)$ is connected, $B-V(P)$ contains an infinite path. Hence, $B-V(P)$ contains a path $R$ from $V\left(C_{i}\right)$ to $V\left(C_{j}\right)$ for some $i$ and $j$ with $j-i \geq 4$. Since $R$ is finite, $R \subset I\left(C_{l}\right)$ for some $l$. By (1), $R \cap P_{n_{k}}=\emptyset$ for all sufficiently large $n_{k}$. Hence, $R$ is contained in a $P_{n_{k}}$-bridge $B_{n_{k}}$ of $I\left(C_{n_{k}}\right)$ for some sufficiently large $n_{k}$. Since $R \cap C_{s} \neq \emptyset$ and $P_{n_{k}} \cap C_{s} \neq \emptyset$ for all $s$ with $i \leq s \leq j, B_{n_{k}}$ has at least four attachments on $P_{n_{k}}$, contradicting the fact that $P_{n_{k}}$ is a $C$-Tutte path in $I\left(C_{n_{k}}\right)$. Hence $B$ is finite. This completes the proof of $(2)$.

By (2), $B \subset I\left(C_{l}\right)$ for some $l$. By (1), B is a $P_{n_{k}}$-bridge of $I\left(C_{n_{k}}\right)$ for all sufficiently large $n_{k}$. Therefore $B$ has at most three attachments on $P$, and if $B$ contains an edge of $C$ then $B$ has just two attachments on $P$. Hence $P$ is a 2 -way infinite $C$-Tutte path in $G$.

We now state and prove the main result of this section which immediately implies (1.3).

(3.4) Theorem. Let $G$ be an infinite 2-connected plane graph with a radial net, let $C$ 
be a facial cycle of $G$, and let $e \in E(C)$. Assume that $G$ is $(4, C)$-connected. Then $G$ contains a 2-way infinite $C$-Tutte path through $e$.

Proof: First, we use (2.4) to construct an infinite sequence $\left(\left(G_{i}, C_{i}, u_{i}, v_{i}, w_{i}\right): i=\right.$ $1,2, \cdots)$. Let $G_{1}=G, C_{1}=C$, let $u_{1}, v_{1}$ be the vertices of $G$ incident with $e$, and let $w_{1}$ be the neighbor of $v_{1}$ in $C$ with $w_{1} \neq u_{1}$. (Note that $w_{1}$ does not play any role in this proof, and it is defined only for the sake of consistency.) Suppose we have constructed $\left(G_{i}, C_{i}, u_{i}, v_{i}, w_{i}\right)$ for some positive integer $i \geq 1$, where $G_{i}$ is an infinite plane graph with a radial net, $C_{i}$ is a facial cycle of $G_{i}, u_{i} v_{i} w_{i}$ is a path in $C_{i}$, and $G_{i}$ is $\left(4, C_{i}\right)$-connected. Since $G_{i}$ admits a radial net, there is a cycle $C_{i}^{*}$ in $G_{i}$ such that $C_{i} \cap C_{i}^{*}=\emptyset$ and $C_{i} \subset I_{G_{i}}\left(C_{i}^{*}\right)$. By applying (2.4) (with $G_{i}, C_{i}, u_{i}, v_{i}$ as $G, C, u, v$ in (2.4), respectively), we find $G_{i+1}, C_{i+1}, u_{i+1}, v_{i+1}, w_{i+1}$ (as $G^{\prime}, C^{\prime}, u^{\prime}, v^{\prime}, w^{\prime}$ in (2.4), respectively). More precisely, there exist a plane graph $G_{i+1}$, a facial cycle $C_{i+1}$ of $G_{i+1}$, and a path $u_{i+1} v_{i+1} w_{i+1}$ in $C_{i+1}$ such that

(1) $G_{i+1}$ is $\left(4, C_{i+1}\right)$-connected and $G_{i+1}-v_{i+1}$ is 2-connected,

(2) $G_{i+1}-\left\{u_{i+1} v_{i+1}, v_{i+1} w_{i+1}\right\} \subset G_{i}$, and no edge of $G_{i}$ joins a vertex of $G_{i+1}-$ $V\left(C_{i+1}\right)$ to a vertex of $G_{i}-V\left(G_{i+1}\right)$,

(3) $\left(G_{i}+\left\{u_{i+1} v_{i+1}, v_{i+1} w_{i+1}\right\}\right)-\left(V\left(G_{i+1}\right)-V\left(C_{i+1}\right)\right)$ is finite and has a plane representation with $C_{i}$ and $C_{i+1}$ as facial cycles,

(4) $v_{i+1} \neq v_{i}$ and $\left(C_{i+1}-v_{i+1}\right) \cap C_{i}=\emptyset$, and

(5) for any subgraph $X$ of $G_{i+1}$ with $C_{i+1} \subset X$, and for any $C_{i+1}$-Tutte path $P_{i+1}$ in $X$ through $u_{i+1} v_{i+1}$ and $w_{i+1}$, there is a $C_{i}$-Tutte path $P_{i}$ of $G_{i}-\left(V\left(G_{i+1}\right)-V(X)\right)$ through $u_{i} v_{i}$ such that $P_{i+1}-v_{i+1} \subset P_{i}$ and, for any $z \in V\left(P_{i}\right)-V\left(P_{i+1}\right)$, either $z \notin V(X)$ or $z \in V(Z)$ for some $P_{i+1}$-bridge $Z$ of $X$ containing an edge of $C_{i+1}$.

Since $G_{i}$ admits a radial net and because $G_{i}-V\left(G_{i+1}\right)$ is finite, it is easy to see that $G_{i+1}$ also admits a radial net. Therefore, by (1), the above construction can be continued with $i+1$ replacing $i$, and we produce the desired infinite sequence $\left(\left(G_{i}, C_{i}, u_{i}, v_{i}, w_{i}\right)\right.$ : $i=1,2, \cdots)$.

Let $G^{\prime}=G+\left\{u_{i} v_{i}, v_{i} w_{i}: i=1,2, \cdots\right\}$. By the first part of $(2), G^{\prime}=\bigcup_{i=1}^{\infty} G_{i}$. By (4), $C_{i} \cap C_{i+2}=\emptyset$ for all $i \geq 1$. By (3), for each $i \geq 1$, we can draw $\left(G_{i}+\right.$ $\left.\left\{u_{i+1} v_{i+1}, v_{i+1} w_{i+1}\right\}\right)-\left(V\left(G_{i+1}\right)-V\left(C_{i+1}\right)\right)$ as a plane graph so that $C_{i+1}$ is its outer 
cycle and $C_{i}$ is a facial cycle. Therefore, $G^{\prime}$ has a plane representation and admits a radial net $N=\left(C_{1}, C_{3}, C_{5}, \ldots\right)$.

For $1 \leq i \leq n$, let $F_{n, i}=G_{i}-\left(V\left(G_{n}\right)-V\left(C_{n}\right)\right)$. Note that $F_{n, i}-\left\{u_{i} v_{i}, v_{i} w_{i}\right\} \subset G \subset$ $G^{\prime}$, and $F_{n, 1} \subset G \subset G^{\prime}$. We will show that for every $n \geq 1, F_{n, 1}$ contains a Tutte path $P_{n, 1}$ through $u v$ such that each component of $P_{n, 1}$ is an $N$-forward path in $G$. To do so, we need to prove a more general result about $F_{n, i}$. For convenience, let $D_{j}:=C_{2 j-1}$ for $j=1,2, \ldots$, and so, $N=\left(D_{1}, D_{2}, D_{3}, \ldots\right)$.

Claim. For all integers $n$ and $i$ with $n \geq i \geq 1, F_{n, i}$ contains a $C_{i}$-Tutte path $P_{n, i}$ between two vertices of $C_{n}$ and through $u_{i} v_{i}$ such that (i) $\left\{u_{n}, v_{n}, w_{n}\right\} \subset V\left(P_{n, i}\right)$, and if $n \geq i+1$ then $\left\{u_{j}, v_{j}, w_{j}: j=i+1, \ldots, n\right\} \subset V\left(P_{n, i}\right)$, and (ii) each component of $P_{n, i}-u_{i} v_{i}$ is an $N$-forward path in $G^{\prime}$.

We use induction on $n-i$. First, assume $n-i=0$. Then $F_{n, i}=C_{i}=C_{n}$. Let $f \in E\left(C_{i}-v_{i}\right)$, and let $P_{n, i}=C_{i}-f$. Then $P_{n, i}$ is a path in $F_{n, i}$ between two vertices of $C_{n}$ and through $u_{i} v_{i}=u_{n} v_{n}$ and $w_{n}$. Hence, $\left\{u_{n}, v_{n}, w_{n}\right\} \subset V\left(P_{n, i}\right)$, and so, (i) holds. Note that $P_{n, i}$ is a $C_{i}$-Tutte path in $F_{n, i}$ (because $F_{n, i}$ has only one $P_{n, i}$-bridge which is induced by the edge $f$ ). Since $P_{n, i} \subset C_{i}$, each component of $P_{n, i}-u_{i} v_{i}$ is (trivially) an $N$-forward path in $G^{\prime}$. Hence (ii) holds.

Now assume that $n-i>0$ and let $P_{n, i+1}$ be a $C_{i+1}$-Tutte path in $F_{n, i+1}$ between two vertices of $C_{n}$ and through $u_{i+1} v_{i+1}$ such that (i) $\left\{u_{n}, v_{n}, w_{n}\right\} \subset V\left(P_{n, i+1}\right)$, and if $n \geq i+2$ then $\left\{u_{j}, v_{j}, w_{j}: j=i+2, \ldots, n\right\} \subset V\left(P_{n, i+1}\right)$, and (ii) each component of $P_{n, i+1}-u_{i+1} v_{i+1}$ is an $N$-forward path in $G^{\prime}$. We wish to apply (5) above. So let $X:=F_{n, i+1}$; then $F_{n, i}=G_{i}-\left(V\left(G_{i+1}\right)-V(X)\right)$. Note that $w_{i+1} \in V\left(P_{n, i+1}\right)$. (For otherwise, $w_{i+1}$ is contained in a $P_{n, i+1}$-bridge of $F_{n, i+1}$ with just two attachments, and one of these attachments is $v_{i+1}$. But then $F_{n, i+1}-v_{i+1}$ is not 2-connected, and so, $G_{i+1}-v_{i+1}$ is not 2-connected, contradicting (1).) Therefore, by (5) (with $P_{n, i+1}, P_{n, i}$ as $P_{i+1}, P_{i}$ in (5), respectively), we see that $F_{n, i}$ contains a $C_{i}$-Tutte path $P_{n, i}$ through $u_{i} v_{i}$ such that $P_{n, i+1}-v_{i+1} \subset P_{n, i}$ and, for any $z \in V\left(P_{n, i}\right)-V\left(P_{n, i+1}\right)$, either $z \notin V\left(F_{n, i+1}\right)$ or $z \in V(Z)-V\left(P_{n, i+1}\right)$ for some $P_{n, i+1}$-bridge $Z$ of $F_{n, i+1}$. Hence $P_{n, i}$ is a path in $F_{n, i}$ between two vertices of $C_{n}$ and through $u_{i} v_{i}$. Clearly, (i) holds.

It remains to show that each component of $P_{n, i}-u_{i} v_{i}$ is an $N$-forward path in $G^{\prime}$. Let $L$ be a component of $P_{n, i}-u_{i} v_{i}$. Let $a, b, c \in V(L)$ such that $b \in V(a L c)$ and $\{a, c\} \subset V\left(D_{k}\right)=V\left(C_{2 k-1}\right)$ for some integer $k \geq 0$. We need to show that $b \notin V\left(D_{j}\right)=$ $V\left(C_{2 j-1}\right)$ for all $j \geq k+2$. Let $L^{\prime}$ denote the component of $P_{n, i+1}-u_{i+1} v_{i+1}$ such that 
$L^{\prime}-v_{i+1} \subset L$

If $\{a, c\} \subset V\left(P_{n, i+1}\right)$, then $\{a, c\} \subset V\left(L^{\prime}\right)$. Hence $b \notin V\left(D_{j}\right)$ for all $j \geq k+2$ because $L^{\prime}$ is an $N$-forward path in $G^{\prime}$ (by induction hypothesis).

If $\{a, c\} \subset V\left(P_{n, i}\right)-\left(V\left(P_{n, i+1}\right)-\left\{v_{i+1}\right\}\right)$, then $a L c \subset P_{n, i}-\left(V\left(P_{n, i+1}\right)-\left\{v_{i+1}\right\}\right)$. Hence, $\{a, c\} \subset V\left(C_{i}\right)$ or $\{a, c\} \subset V\left(C_{i+1}\right)$, and so, $D_{k}=C_{i}$ or $D_{k}=C_{i+1}$. Also $b=v_{i+1}$ or $b \notin V\left(P_{n, i+1}\right)$. If $b=v_{i+1}$, then by $(4), b \notin V\left(C_{l}\right)$ for all $l \geq i+2$, and so, $b \notin V\left(C_{2 j-1}\right)=V\left(D_{j}\right)$ for all $j \geq k+2$. So assume that $b \notin V\left(P_{n, i+1}\right)$. Then by (5), either $b \notin V\left(F_{n, i+1}\right)$ or $b \in V(Z)$ for some $P_{n, i+1}$-bridge $Z$ of $F_{n, i+1}$ containing an edge of $C_{i+1}$. If $b \notin V\left(F_{n, i+1}\right)$ then $b \notin V\left(C_{l}\right)$ for all $l \geq i+2$; and if $b \in V(Z)$ then by planarity, $b \notin V\left(C_{l}\right)$ for all $l \geq i+2$. Again, $b \notin V\left(C_{2 j-1}\right)=V\left(D_{j}\right)$ for all $j \geq k+2$.

So assume by symmetry that $a \notin V\left(P_{n, i+1}\right)$ and $c \in V\left(P_{n, i+1}\right)-\left\{v_{i+1}\right\}$. Hence $c \notin V\left(C_{i}\right)$, and so, $a \notin V\left(C_{i}\right)$. Because $a \notin V\left(P_{n, i+1}\right)$ and by (5), either $a \notin V\left(F_{n, i+1}\right)$ or $a \in V(Z)$ for some $P_{n, i+1}$-bridge $Z$ of $F_{n, i+1}$ containing an edge of $C_{i+1}$, and so, $a \notin V\left(C_{l}\right)$ for all $l \geq i+2$. Hence $\{a, c\} \subset V\left(C_{i+1}\right)$, and so, $D_{k}=C_{i+1}$. Suppose $b \notin V\left(P_{n, i+1}\right)-\left\{v_{i+1}\right\}$. Then by $(5)$, either $b \notin V\left(F_{n, i+1}\right)$, or $b=v_{i+1}$, or $b \in V(Z)$ for some $P_{n, i+1}$-bridge $Z$ of $F_{n, i+1}$ containing an edge of $C_{i+1}$. Hence $b \notin V\left(C_{l}\right)$ for all $l \geq i+2$, and so, $b \notin V\left(C_{2 j-1}\right)=V\left(D_{j}\right)$ for all $j \geq k+2$. So assume that $b \in V\left(P_{n, i+1}\right)-\left\{v_{i+1}\right\}$. Hence, there is some $b^{\prime} \in V\left(C_{i+1}\right) \cap V\left(L^{\prime}\right)$ such that $b \in$ $V\left(c L^{\prime} b^{\prime}\right)$. Therefore, $\left\{b^{\prime}, c\right\} \subset V\left(C_{i+1}\right)=V\left(D_{k}\right)$. Since $L^{\prime}$ is an $N$-forward path in $G^{\prime}$, $b \notin V\left(C_{2 j-1}\right)=V\left(D_{j}\right)$ for all $j \geq k+2$.

Therefore $L$ is an $N$-forward path in $G^{\prime}$. Hence $P_{n, i}$ gives the desired path in the claim.

By the above claim, $F_{n, 1}$ contains a $C_{1}$-Tutte path $P_{n}:=P_{n, 1}$ between two vertices of $C_{n}$ and through $e=u_{1} v_{1}$ such that each component of $P_{n}-e$ is an $N$-forward path in $G^{\prime}$. (Note that $P_{n} \subset G$ because $F_{n, 1} \subset G$.) Since $\left\{u_{i}, v_{i}, w_{i}: i=2, \cdots, n\right\} \subset V\left(P_{n}\right)$ for all $n \geq 1, P_{2 n-1}$ is also a $C_{1}$-Tutte path in $I_{G^{\prime}}\left(C_{2 n-1}\right)=I_{G^{\prime}}\left(D_{n}\right)$ between two vertices of $D_{n}$ and through $e$. Hence, by (3.3), $\left\{P_{2 n-1}\right\}$ has a subsequence $\left\{P_{n_{k}}\right\}$ converging to a 2-way infinite $C_{1}$-Tutte path $P$ in $G^{\prime}$ through $e$. Since $P_{n_{k}} \subset G$ for all $n_{k}, P$ is a 2-way infinite $C$-Tutte path in $G$ through $e$. 


\section{References}

[1] N. Dean, R. Thomas and X. Yu, Spanning paths in infinite planar graphs, J. Graph Theory 23 (1996) 163-174.

[2] C. St. J. A. Nash-Williams, Hamiltonian lines in infinite graphs with few vertices of small valency, Aequationes Math. 7 (1971) 59-81.

[3] C. St. J. A. Nash-Williams, Unexplored and semi-explored territories in graph theory in New Directions in Graph Theory (ed. F. Harary), Academic Press (1973) 149-186.

[4] R. Thomas and X. Yu, 4-Connected projective-planar graphs are hamiltonian, $J$. Combin. Theory Ser. B 62 (1994) 114-132.

[5] C. Thomassen, Infinite graphs in Selected Topics in Graph Theory Vol. 2 Academic Press (1983) 129-160.

[6] C. Thomassen, A theorem on paths in planar graphs, J. Graph Theory 7 (1983) $169-176$.

[7] T. Tutte, A theorem on planar graphs, Trans. Amer. Math. Soc. 82 (1956) 99-116.

[8] H. Whitney, A theorem on graphs, Ann. of Math. 32 (1931) 378-390. 